\title{
Vortices and Flat Connections
}

\author{
S. M. Nasir* \\ Department of Applied Mathematics and Theoretical Physics \\ University of Cambridge \\ Silver Street, Cambridge CB3 9EW, England
}

July, 1998

\begin{abstract}
At Bradlow's limit, the moduli space of Bogomol'nyi vortices on a compact Riemann surface of genus $g$ is determined. The Kähler form, and the volume of the moduli space is then computed. These results are compared with the corresponding results previously obtained for a general vortex moduli space.
\end{abstract}

1. The Abelian Higgs model in $(2+1)$ dimensions is an interesting arena to study vortices. The coupling constant of the model determines the nature of interactions among vortices. At the critical coupling, the model admits static and finite energy Bogomol'nyi vortex solutions [1]. Stability of these solutions is ensured by topology. We will consider vortices in a space-time of the form $\mathbf{R} \times M$, where $M$ is a compact two dimensional manifold. The metric of the space-time is taken to be $d s^{2}=d x_{0}^{2}-\Omega\left(x_{1}, x_{2}\right)\left(d x_{1}^{2}+d x_{2}^{2}\right)$, where $x_{1}$ and $x_{2}$ denote local coordinates on $M$. Let $A_{\mu}, \quad(\mu=0,1,2)$ be a $U(1)$ gauge potential and $\phi$ be a complex scalar field. Working in the gauge $A_{0}=0$, the Lagrangian of the model at the critical coupling is $L=T-V$, where

$$
T=\frac{1}{2} \int_{M} d^{2} x\left(\dot{A}_{i} \dot{A}_{i}+\Omega \dot{\phi} \dot{\bar{\phi}}\right), \quad(i=1,2)
$$

$$
\begin{aligned}
V=\frac{1}{2} \int_{M} d^{2} x \Omega & {\left[\frac{1}{2} F_{i j} F^{i j}+D_{i} \phi \overline{D^{i} \phi}\right.} \\
& \left.+\frac{1}{4}\left(|\phi|^{2}-1\right)^{2}\right]
\end{aligned}
$$

are respectively, the kinetic and the potential energies. Here, $D_{i}=\partial_{i}-i A_{i}$, and $F_{12}=$ $\partial_{1} A_{2}-\partial_{2} A_{1}$, is the magnetic field. The following first order Bogomol'nyi vortex equations are obtained by minimizing the potential energy

$$
\begin{gathered}
\left(D_{1}+i D_{2}\right) \phi=0 \\
F_{12}+\frac{\Omega}{2}\left(|\phi|^{2}-1\right)=0 .
\end{gathered}
$$

The above equations admit static multi-vortex solutions. The solutions are parametrized by a $2 N$ dimensional moduli space, $M_{N}$, where $N$ is the number of zeros of the Higgs field counted with multiplicity [2, 3]. $N$ - called the vortex number - is related to the total magnetic flux by $N=\frac{1}{2 \pi} \int_{M} d^{2} x F_{12}$. The potential energy of a configuration of static $N$-vortices is $\pi N$.

*e-mail address: S.M.Nasir@damtp.cam.ac.uk 
Topologically, $M_{N}$ is just the symmetrized $N$ th power of $M$. The moduli space has a natural Riemannian metric induced from the kinetic energy expression (1). This is given by

$$
d s^{2}=\frac{1}{\pi} \int_{M} d^{2} x\left(\delta A_{i} \delta A_{i}+\Omega \delta \phi \delta \bar{\phi}\right), \quad(i=1,2) .
$$

In obtaining (5) from (1) we have multiplied (1) by $2 / \pi$ to agree with the conventions of [4]. It is to be noted that there is a Gauss' law constraint arising from the equation of motion of $A_{0}$. This constraint ensures that $M_{N}$ consists of the solutions of eqns. $(3,4)$ modulo gauge transformations connected to the identity. Hereafter, by 'gauge transformations' we will mean those gauge transformations connected to the identity. Through Manton's work [5] it is known that at low energy - when most of the degrees of freedom remain unexcited the moduli space can be used to describe interesting physical phenomena associated with vortices, such as scattering [6], thermodynamics [4, 7, 8], and the phase transition of vortices at near-critical coupling [9], etc. In the moduli space approximation the vortex dynamics can be thought of as geodesic motion on the moduli space.

For a compact surface, in order for vortex solutions to exist one has to satisfy Bradlow's bound [10]. The bound is $4 \pi N \leq A$, where $A$ is the area of $M$. This can be obtained by integrating (4) over $M$, and noticing that the integral of $|\phi|^{2}$ over $M$ is positive. The bound means that for a given area there is a limit on the number of vortices one can have on $M$. At Bradlow's limit, $A=4 \pi N$, the Higgs field $\phi$ must vanish everywhere on $M$. Then, the Bogomol'nyi equations reduce to the following single equation

$$
F_{12}=\frac{\Omega}{2}
$$

This is an equation for a constant magnetic field on $M$. The energy of the configuration is still $\pi N$.

It might be thought that (6) has a unique solution up to gauge equivalence, which in turn will mean that the moduli space is just a point. However, if $M$ has non-contractible loops (onecycles), i.e. if the first homotopy group of $M$ is non-trivial, then the moduli space of solutions of (6) is non-trivial as well. A solution of (6) up to gauge equivalence is given, in addition to a gauge potential that solves (6), by specifying holonomies around a basis of onecycles. The holonomies around any two homologous loops are generally different as the magnetic field is non-zero on $M$, but the difference can be completely determined by using Stokes' theorem. Now, linearizing (6) around a particular solution one can see that the perturbed gauge potentials satisfy the equations for flat $U(1)$ connections for which the magnetic field is zero. Flat connections are associated with large gauge transformations. Flat connections up to gauge equivalence are also given by specifying their holonomies around a basis of onecycles. Hence, when $\phi=0$ the moduli space of Bogomol'nyi vortices is no longer $M_{N}$. Locally, the tangent space of the vortex moduli space at Bradlow's limit can be identified with the tangent space of the space of flat $U(1)$ connections on $M$. With an abuse of notation, in future the moduli space of solutions of (6) and the space of flat $U(1)$ connections will be denoted by $M_{f}$. It was demonstrated long ago by Aharonov and Bohm [11] that flat connections play quite a non-trivial role in quantum physics. It should be noted that flat connections do not contribute to the moduli of the Bogomol'nyi equations when the Higgs field is non-vanishing. 
In passing we would like to point out that when $\phi=0$, one may consider $F_{12}=0$ as solutions of the static Abelian Higgs model. The energy is then $A / 8$ with no restriction on the value of $A$. However, these solutions cannot be obtained from Bogomol'nyi equations. Henceforth, we will only consider vortices at Bradlow's limit, and $M$ is taken to be of genus $g \geq 1$. 2. Let $A=A_{1} d x_{1}+A_{2} d x_{2}$ solve (6). Then, $A+\delta A$, where $\delta A=a_{1} d x_{1}+a_{2} d x_{2}$ is a flat connection, is also a solution of (6). The equation satisfied by $\delta A$ is $d \delta A=0$. The metric on $M_{f}$ is obtained by putting $\phi=0$ in (5). This is

$$
d s^{2}=\frac{1}{\pi} \int_{M} d^{2} x\left[\left(a_{1}\right)^{2}+\left(a_{2}\right)^{2}\right] .
$$

Notice that the metric of $M$ does not appear in the above expression. This means that the metric information of $M$ is not carried over to $M_{f}$. One can see that $M_{f}$ inherits a complex structure from $M$. The map $\mathcal{I}$ given by $\mathcal{I}: a_{j} \rightarrow-\epsilon_{j k} a_{k}$, where $\epsilon_{j k}$ is the antisymmetric tensor with $\epsilon_{12}=1$, leaves invariant (7) and the equation for a flat connection. Moreover, $\mathcal{I}^{2}=-\mathbf{1}_{2}$. Hence, $\mathcal{I}$ defines an almost complex structure on $M_{f}$. This almost complex structure can be used to define the following $(1,1)$ form on $M_{f}$

$$
\omega(\delta A, \delta B)=\frac{1}{\pi} \int_{M}\left(\delta A_{z} \wedge \overline{\delta B_{z}}-\delta B_{z} \wedge \overline{\delta A_{z}}\right) .
$$

Here, we have used complex coordinates $(z=$ $\left.x_{1}+i x_{2}\right)$, and $\delta A=\left(\delta A_{z}+\right.$ c.c $)$. Clearly, $\omega$ is manifestly real. Using $d \delta A=d \delta B=0$, it can also be shown that $\omega$ is a closed form. Thus, $\mathcal{I}$ is a complex structure and $\omega$ defines a Kähler form. Among many uses of this Kähler form one can, for example, compute the volume of $M_{f}$. Recently, we obtained an expression for the Kähler form on $M_{N}$ and also, we computed the volume of $M_{N}[\mathbb{8}$. We will see that when
$A=4 \pi N$, the Kähler form on $M_{f}$ gets mapped to the Kähler form on $M_{N}$.

Although a known fact, in order to setup the stage for the main part of this paper we intend to show that the space of flat $U(1)$ connections on $M$ modulo gauge transformations, i.e. $M_{f}$, is parametrized by the Picard variety, $\tilde{J}$, of $M[\overline{[2]}$. $\tilde{J}$ is dual to the Jacobian $J$ of $M . J$ is a $2 g$-dimensional real torus. Let $\nu_{i}, \quad(i=1, \cdots, 2 g)$ be a basis of $2 g$ one-cycles of $M$. Let $w_{\rho}, \quad(\rho=1, \cdots, g)$ be a basis of $g$ holomorphic one-forms on $M$. Define the period matrix $W=\left(w_{\rho i}\right)$, where $w_{\rho i}=\oint_{\nu_{i}} w_{\rho}, \quad(\rho=1, \cdots, g, \quad i=1, \cdots, 2 g)$. The columns $W_{i},(i=1, \cdots, 2 g)$ of $W$ can be thought of as spanning a $2 g$-dimensional lattice in $\mathbf{C}^{g}$. $J$ is defined as the torus $\mathbf{C}^{g} / W$. Riemann's bilinear relations can be used to normalize the period matrix $W$ [13]. One can choose $W$ such that $\left(W^{t}, \bar{W}^{t}\right)$ is a symmetric matrix with $\operatorname{Im}(W)>0$. Further, the elements of $W$ can be restricted to satisfy $w_{\rho i}=\delta_{\rho i}$ for $\rho=1, \cdots, g$ and $i=1, \cdots, g$, the remaining elements being arbitrary with positive imaginary parts [13]. Let us choose a basis of $2 g$ one-cocycles $\alpha_{i}$ such that $\oint_{\nu_{i}} \alpha_{j}=\delta_{i j}, \quad(i, j=$ $1, \cdots, 2 g)$. These one-cocycles are the $2 g$ generators of the cohomology group $H^{1}(M, \mathbf{Z})$. A canonical basis of $\nu_{i}$ can be chosen such that the cocycles also satisfy $\int_{M} \alpha_{i} \alpha_{j}=\delta_{i, j+g}$, where in the integration wedge product is implied. The $g$ holomorphic one-forms can be expressed in terms of $\alpha_{i}$ as $w_{\rho}=\sum_{i=1}^{2 g} w_{\rho i} \alpha_{i}$ for $(\rho=1, \cdots, g)$. Reciprocally, $\alpha_{i}$ can be expressed in terms of $w_{\rho}$ as $\alpha_{i}=\sum_{\rho=1}^{g}\left(\gamma_{i \rho} w_{\rho}+c . c\right)$, for $(i=1, \cdots, 2 g)$ where the matrix $\Gamma=\left(\gamma_{\rho i}\right)$ is required to satisfy

$$
\Gamma^{t} W+\overline{\Gamma^{t}} \bar{W}=\mathbf{1}_{2 g}
$$


We note that $\tilde{J}$ is defined as the torus $\mathbf{C}^{g} / \Gamma$. Hence, $\tilde{J}$ is dual to $J$.

Let $c_{\rho},(\rho=1, \cdots, g)$ denote complex coordinates on $M_{f}$ (that $M_{f}$ is $2 g$-dimensional will be evident below). Then a real flat connection $A_{f}$ (modulo gauge transformations) can be expressed as

$$
A_{f}=2 \pi \sum_{\rho=1}^{g}\left(c_{\rho} w_{\rho}+c . c\right) .
$$

Imposing Gauss' law on the flat connections one can see that the above is the most general expansion for a flat connection. The holonomy, $h_{j}$, of $A_{f}$ around a one-cycle $\nu_{j}$ is given by

$h_{j}=\exp \left(i \oint_{\nu_{j}} A_{f}\right)=\exp \left(2 \pi i \sum_{\rho=1}^{g}\left(c_{\rho} w_{\rho j}+c . c\right)\right)$.

As noted earlier, for a complete specification of $A_{f}$ one needs to specify all of the holonomies $h_{j}$. Eqn.(11) implies that $h_{j}$ is periodic with the period matrix being $\Lambda=\left(\lambda_{\rho i}\right), \quad(\rho=$ $1, \cdots, g, \quad i=1, \cdots, 2 g)$, say. Then the $2 g$ columns of $\Lambda$ span a $2 g$-dimensional lattice in $\mathbf{C}^{g}$. Thus, $M_{f}$ is parametrized by a $2 g$ dimensional real torus $\mathbf{C}^{g} / \Lambda$. Further, the following relation for $\Lambda$ is implied by (11)

$$
\Lambda^{t} W+\overline{\Lambda^{t}} \bar{W}=\mathbf{1}_{2 g} .
$$

Comparing the above equation with (9) one gets $\Lambda=\Gamma$. This identifies $M_{f}$ with $\tilde{J}$.

There are $g$ independent holomorphic oneforms on $M_{f}$. Using $c_{\rho}$ as coordinates of $M_{f}$, these are given by $d c_{\rho},(\rho=1, \cdots, g)$. A basis of one-cycles in $M_{f}$ is given by the $2 g$ lines $t \Lambda_{i}, 0 \leq t \leq 1$, with $\Lambda_{i}$ identified with 0 to produce a closed loop. Then from the discussion preceeding (9), one deduces that the $2 g$ generators of $H^{1}\left(M_{f}, \mathbf{Z}\right)$ are given by $\tilde{\xi}_{i}=$ $\sum_{\rho=1}^{g}\left(w_{\rho i} d c_{\rho}+\right.$ c.c $)$. Now from (8), we get the fol- lowing Kähler form on $M_{f}$

$$
\tilde{\omega}=4 \pi \sum_{\rho, \rho^{\prime}, i=1}^{g}\left(w_{i \rho} \bar{w}_{i+g \rho^{\prime}}-\bar{w}_{i \rho^{\prime}} w_{i+g \rho}\right) d c_{\rho} \wedge d \bar{c}_{\rho^{\prime}}
$$

In terms of $\tilde{\xi}_{i}, \tilde{\omega}$ can be written as

$$
\tilde{\omega}=4 \pi \sum_{i=1}^{g} \tilde{\xi}_{i} \tilde{\xi}_{i+g}
$$

In obtaining the above from (13) we have used the Riemann bilinear relations. The volume of $M_{f}$ is then

$$
\mathrm{Vol}_{f}=\frac{1}{g !} \int_{M_{f}} \tilde{\omega}^{g}=(4 \pi)^{g}
$$

where use has been made of the fact that $\int_{M_{f}} \prod_{i=1}^{g} \tilde{\xi}_{i} \tilde{\xi}_{i+g}=1$. It is useful to notice that the volumes of $J$ and $\tilde{J}$ are the same. The computation of the volume of the space of flat connections on a compact Riemann surface is not new. For $S U(2)$ and $S O(3)$ Yang-Mills theory, Witten [14] computed the volume of the space of flat connections by a remarkable use of the Verlinde formula [15] in conformal field theory. 3. The general formula for the Kähler form on the vortex moduli space $M_{N}$, when $\phi$ is nonvanishing, is [8]

$$
\omega=(A-4 \pi N) \eta+4 \pi \sum_{i=1}^{g} \xi_{i} \xi_{i+g}
$$

where $\eta$ is an area form on $M_{N}$ normalized to unity and $\xi_{i},(i=1, \cdots, 2 g)$ are the $2 g$ generators of $H^{1}\left(M_{N}, \mathbf{Z}\right)$. When $\phi=0$ is zero, i.e. $A=4 \pi N$, the Kähler form in (16) reduces to

$$
\omega=4 \pi \sum_{i=1}^{g} \xi_{i} \xi_{i+g}
$$

At this point we should remind the reader that this Kähler form is defined on $M_{N}$, not on $\tilde{J}$ whose tangent space coincides with the tangent space of the moduli space of vortices when $\phi=$ 
0. However, it can be shown that the Kähler form $\omega$ on $M_{N}$ is mapped in a one-to-one way to the Kähler form $\tilde{\omega}$ on $J$. As $\tilde{J}$ is isomorphic to $J$ it is enough to establish an isomorphism between $H^{1}\left(M_{N}, \mathbf{Z}\right)$ and $H^{1}(J, \mathbf{Z})$. First, notice that Jacobi's inversion theorem [13] implies that there is an isomorphism between $J$ and $M_{g}$ where $M_{g}$ is the moduli space of $g$ vortices, and $g$ is the genus of $M$. This implies the isomorphism between $H^{1}(J, \mathbf{Z})$ and $H^{1}\left(M_{g}, \mathbf{Z}\right)$. Next, using the Lefschetz hyperplane section theorem [13], one sees that there is an isomorphism between $H^{1}\left(M_{N}, \mathbf{Z}\right)$ and $H^{1}(J, \mathbf{Z})$ for $N \geq g$ and $g>1$. The isomorphism for other values of $N$ and $g$ can also be easily established by arguments used in [16].

It is of interest to see if one can relate $\mathrm{Vol}_{f}$ to the volume of $M_{N}$ near Bradlow's limit when $\epsilon=A-4 \pi N$ is a small positive quantity. For genus $g \geq 1$ and $N \geq 2 g-1, M_{N}$ has a bundle structure, where the base is $J$, and the fibre is $C P_{N-g}$. For $N \leq g, M_{N}$ is analytically homeomorphic to a $2 N$-dimensional submanifold of the Jacobian. Generically, the volume of $M_{N}$ is not just a product of the volume of the base and the volume of the fibre. The volume of $M_{N}$ as computed in 8 is

$\mathrm{Vol}_{N}=(A-4 \pi N)^{N-g} \sum_{i=0}^{g}\left(\frac{(4 \pi)^{i}(A-4 \pi N)^{g-i} g !}{(N-i) !(g-i) ! i !}\right) \cdot[1$

In this formula $N \geq g$. It is easy to write an analogous formula for $N<g$. Near Bradlow's limit the above volume can be written as

$$
\mathrm{Vol}_{N}=(4 \pi)^{g} \frac{\epsilon^{N-g}}{(N-g) !}+O\left(\epsilon^{N-g+1}\right) .
$$

Neglecting the higher order corrections the above can be written as

$$
\mathrm{Vol}_{N}=\mathrm{Vol}_{f} \times \frac{\epsilon^{N-g}}{(N-g) !}
$$

where the factor $\epsilon^{N-g} /(N-g)$ ! can be thought of as a contribution coming from the fibre $C P_{N-g}$. Indeed, using (16) and the cohomolgy class of the fibre one can show that the volume of the fibre is $(A-4 \pi N)^{N-g} /(N-g)$ !.

In conclusion, we would like to clarify the following apparent puzzle. In computing the Kähler form (16), one needs to extract the nonsingular parts of the expressions like $\partial_{z} \log |\phi|^{2}$ around the zeros of $\phi$. This, however, does not invalidate the derivation of (16) when $\phi=0$ as one may think. From the Bogomol'nyi equations one can always express $\partial_{z} \log |\phi|^{2}$ in terms of the gauge potentials, which can in principle be used to derive (16) regardless of whether $\phi$ is zero or not.

Acknowledgements: I would like to thank my supervisor Dr N. S. Manton for his continuous guidance and very helpful discussions. Also thanks to P. Irwin for critical comments on this manuscript. This work was supported by the Overseas Research Council, the Cambridge Commonwealth Trust and Wolfson college.

\section{References}

E. B. Bogomol'nyi, Sov. J. Nucl. Phys. 24 (1976) 449.

[2] C. H. Taubes, Comm. Math. Phys. 72 (1980) 277.

[3] A. Jaffe and C. H. Taubes, Vortices and Monopoles (Birkhäuser, Boston, 1980).

[4] N. S. Manton, Nucl. Phys. B 400 (1993) 624.

[5] N. S. Manton, Phys. Lett. B 110 (1982) 54. 
[6] T. M. Samols, Comm. Math. Phys. 145 149 (1992); Ph.D Thesis, (Cambridge University 1990) Unpublished.

[7] P. A. Shah and N. S. Manton, J. Math. Phys. 35 (1994) 1171.

[8] N. S. Manton and S. M. Nasir, To appear in Comm. Math. Phys. hep-th/9807017

[9] P. A. Shah, Nucl. Phys. B 438 (1995) 589

[10] S. Bradlow, Comm. Math. Phys. 135 (1990) 1.

[11] Y. Aharonov and D. Bohm, Phys. Rev. 115 (1959) 485.
[12] R. C. Gunning, Lectures on Riemann Surfaces (Princeton University Press, New Jersey, 1966).

[13] P. Griffiths and J. Harris, Principles of Algebraic Geometry (John Wiley \& Sons, Inc., New York, 1978).

[14] E. Witten, Comm. Math. Phys. 141 (1991) 153.

[15] E. Verlinde, Nucl. Phys. B 300 (1988) 360.

[16] I. G. MacDonald, Topology 1 (1962) 319. 